\title{
Límites de la espacialidad de situación para la descripción merleaupontiana del fenómeno del movimiento y el aporte de la noción de postura
}

\author{
Ariela Battán Horenstein \\ Universidad Nacional de Córdoba/CONICET
}

\begin{abstract}
Resumen: El presente trabajo intenta constituir un aporte a la tarea de ganar precisión conceptual en la consideración de las temáticas del espacio y el movimiento humano desde una perspectiva fenomenológica y toma como horizonte teórico las lúcidas reflexiones de Maurice Merleau-Ponty. Se analizan los aportes de la distinción entre una espacialidad de situación y una espacialidad de posición, se revisan de manera crítica las limitaciones de la misma y se propone la noción de postura como un complemento descriptivo que permite hacer extensiva la reflexión merleaupontiana acerca del movimiento a fenómenos motrices de agentes diestros y entrenados.
\end{abstract}

Palabras clave: Merleau-Ponty; cuerpo; movimiento; situación; postura

\begin{abstract}
Situation-Spaciality's Limits for a Merleaupontian Description of the Movement Phenomenon and the Contribution of the Notion of Posture". El presente trabajo intenta constituir un aporte a la tarea de ganar precisión conceptual en la consideración de las temáticas del espacio y el movimiento humano desde una perspectiva fenomenológica y toma como horizonte teórico las lúcidas reflexiones de Maurice Merleau-Ponty sobre estas temáticas. Se analizan en el mismo los aportes de la distinción entre una espacialidad de situación y una espacialidad de posición, se revisan de manera crítica las limitaciones de la misma y se propone la noción de postura como un complemento descriptivo que permite hacer extensiva la reflexión merleaupontiana acerca del movimiento a fenómenos motrices de agentes diestros y entrenados.
\end{abstract}

Keywords: Merleau-Ponty; body; movement; situation; posture

\footnotetext{
* Este trabajo ha sido realizado en el marco del PIP CONICET 114-20110100073, "La posibilidad de fundamentación fenomenológica de la intencionalidad corporal: análisis y descripción de la relación percepción y movimiento", 2012-2014 y del Proyecto de Investigación "Fenomenología del cuerpo y análisis del dolor" (FFI 2013-43240-P). Dirección General de Investigación Científica y Técnica del Ministerio de Economía y Competitividad, España, dirigido por el doctor Agustín Serrano de Haro. Agradezco los acertados y generosos comentarios del doctor Serrano de Haro y del profesor Alejandro Laregina que me permitieron mejorar una primera y provisoria versión del trabajo. He intentado, además, atender a las sugerencias y correcciones de los evaluadores, en virtud de ellas he podido aclarar puntos que de otro modo hubieran permanecido confusos. Mi agradecimiento a los editores de la Revista Areté por su gentileza y generosidad durante el proceso de publicación.
} 


\begin{abstract}
"Te voy a enseñar algo, me sugirió, es una postura preparatoria, que hacemos en el suelo, y la llamamos 'el puente', porque nuestro peso queda suspendido entre la mano izquierda, firmemente plantada en el suelo, y el pie derecho, también plantado en el suelo. Entre estos dos puntos fijos, todo el cuerpo permanece expectante, al acecho, suspendido."
\end{abstract}

(El cuaderno de Bento, John Berger ${ }^{1}$ )

\title{
1. Introducción
}

El presente trabajo intenta constituir un aporte a la tarea de ganar precisión conceptual en la consideración del espacio y el movimiento humano desde una perspectiva fenomenológica y en el horizonte teórico provisto por las lúcidas reflexiones de Maurice Merleau-Ponty. Este filósofo nos ha legado las herramientas necesarias para emprender el estudio del fenómeno de la motricidad y es posible, a lo largo de su obra, encontrar infinidad de testimonios del alto grado de sistematización y desarrollo de conceptos centrales como "intencionalidad motriz", "espacio vivido", "esquema corporal”, entre otros. Muchos trabajos y autores, en distintas épocas de la larga exégesis del pensamiento merleaupontiano, han hecho eco de esos planteos, han repetido, esclarecido y utilizado sus palabras y principales tesis ${ }^{2}$ y, gracias a ellos, la temática de la motricidad humana ha ido adquiriendo relevancia hasta colocarse a la par del interés por el estudio de la percepción. Sin pretender restar valor a esos logros, resulta oportuno proponer como desafio intelectual la tarea de seguir avanzando con Merleau-Ponty, pero con el objetivo de llegar más lejos de lo que este autor lo hizo. Me refiero, concretamente, al desafio de hacer extensivos los análisis y descripciones merleaupontianos a una serie de fenómenos motrices que el filósofo no consideró en su profundidad, ya fuera porque excedian sus intereses o, simplemente, porque le resultaban desconocidos. El desarrollo de

1 John Berger se refiere aquí a la bailarina María Muñoz de la compañía Mal Pelo de España, a quien dibuja en el mencionado libro. Cf. Berger, J., El cuaderno de Bento, Buenos Aires: Alfaguara, 2013, p. 22.

2 Lefort, C., "Le sens de l'orientation", en: Merleau-Ponty, M., Notes de cours sur L'origine de la geometrie de Husserl. Suivi de Recherches sur la phénoménologie de Merleau-Ponty, Barbaras, R. (dir.), París: Épiméthée, PUF, 1989, pp. 221-238; y en particular los trabajos de Renaud Barbaras "Motricité et phénoménalité chez le dernier Merleau-Ponty", en : Richir, M. y E. Tassin (eds.), Merleau-Ponty, Phénoménologie et Expériences, Grenoble: Jérôme Millon, 1992; idem., "Perception and Movement: the End of the Metaphysical Approach", en: Evans, F. y L. Lawlor (eds.), Chiasms: Merleau-Ponty's Notion of Flesh, Albany: State University of New York Press, 2000. También $c f$. Dillon, M., Merleau-Ponty's Ontology, Bloomington e Indianapolis: Indiana University Press, 1988.

ARETÉ Revista de Filosofía, vol. XXVIII, Nº 2, 2016 / ISSN 1016-913X 
la investigación en el ámbito de la fenomenología ha dado lugar a la ampliación de los objetos y fenómenos de estudio de la disciplina. Hoy reclaman nuestra atención el cuerpo, el espacio, el tocar, la acción, la danza, entre otros. En este trabajo me interesa, en particular, reflexionar acerca de las herramientas descriptivas necesarias para la aprehensión del movimiento de precisión de agentes motrices entrenados (bailarines, performers, deportistas, músicos, etcétera) y de agentes que requieren destreza motriz en el uso de instrumentos o de sus cuerpos. Entiendo que este fenómeno del movimiento diestro constituye un objeto nuevo de reflexión, para el cual las consideraciones merleaupontianas sobre el movimiento de agentes normales o patológicos son de gran utilidad, mas no alcanzan para la aprehensión del fenómeno en su complejidad. El pensamiento merleaupontiano goza de una vitalidad y una apertura que no solo habilita, sino también invita, a aceptar el reto de la actualización y la profundización de sus tesis y conclusiones de las décadas de 1940 y 1950, a la luz de los últimos avances en las investigaciones sobre percepción, cognición y motricidad. Son dignos de mencionar, en este sentido, los trabajos recientes de Gallagher, Morris, Sheets-Johnstone y Kristensen ${ }^{3}$, autores que combinan de manera armónica la reflexión sobre el legado y los nuevos fenómenos objeto de análisis. En coincidencia con la motivación que inspira a estos autores, me propongo emprender en este trabajo la revisión crítica de algunas herramientas teóricas centrales de la concepción merleaupontiana de la espacialidad y el movimiento y ofrecer argumentos para la superación de esas críticas. Intentaré, en primer lugar, someter a discusión el par posición-situación -distinción central en el contexto de la reflexión merleaupontiana sobre la motricidad y la espacialidad del cuerpo-, con el objetivo de considerar su aporte y, a la vez, mostrar las limitaciones que este posee cuando se lo aplica a la comprensión de un tipo de movimiento como el de agentes motrices diestros o entrenados. Para esto será necesario revisar, también, la relación entre el espacio espacializado y el espacio espacializante, es decir, entre el espacio comprendido como continente físico de los objetos y el espacio como articulador de relaciones posibles. Estas dos nociones que son presentadas por Merleau-Ponty de manera sumamente escueta en el apartado sobre el espacio de su Fenomenología de la Percepción, constituyen un aporte insoslayable para nuestro trabajo ya que, a diferencia de la espacialidad de

3 Gallagher, S., How the Body Shapes de Mind, Nueva York: Oxford University Press, 2005; Morris, D., The Sense of the Space, Albany: SUNY Press, 2004; Sheets-Johnstone, M., The Primacy of Movement, Amsterdam: John Benjamin, 1999; Kristensen, S., "Le mouvement de la création. Merleau-Ponty et le corps de 1' artiste” en: Alter. Revue de Phénoménologie (2008), pp. 243-260. 
situación y la espacialidad de posición, concebidas como instancias excluyentes y antitéticas, el espacio espacializante y el espacio espacializado se encuentran relacionados y es posible pasar de uno a otro. Finalmente, deseo presentar la noción de postura como un aporte complementario al par posición-situación. La introducción de esta noción permitirá tener en cuenta, en la descripción de los fenómenos motrices, aquellos componentes geométricos del espacio y estados del organismo que la fenomenología merleaupontiana relegaba al dominio del cuerpo y el espacio objetivos y que, por este motivo, no eran considerados en el análisis del fenómeno del movimiento, una vez instituida la dimensión fenomenal. Cabe aclarar que la noción de postura no es ajena al vocabulario merleaupontiano ${ }^{4}$, aunque su significación se encuentra determinada por la concepción del espacio y del movimiento que se intenta revisar críticamente en este trabajo. En consecuencia, me propongo recurrir aquí a la definición de esta noción propuesta por Agustín Serrano de Haro en su obra La precisión del cuerpo. Análisis filosófico de la puntería ${ }^{5}$. Esta definición, según entiendo y tal como intentaré mostrar más adelante, proporciona la clave que se reclama para emprender la descripción de los fenómenos motrices de agentes diestros.

Las conclusiones a las que arribemos en este trabajo, el cual tiene una intención crítica y a la vez propositiva, nos permitirán ampliar el repertorio conceptual de la fenomenología para el estudio de la acción humana en su variedad y diversidad.

\section{Posición y situación en la comprensión del espacio}

Quienes trabajamos desde la perspectiva fenomenológica propuesta por Merleau-Ponty acostumbramos a emprender las descripciones de los fenómenos de manera unidireccional, avanzando desde el ámbito de lo objetivo hacia el de lo vivido, pero pocas veces emprendemos el camino inverso, esto es, el que retorna de lo vivido a lo objetivo. Ciertas experiencias motrices y artísticas

\footnotetext{
4 La noción de postura aparece en vinculación con otra noción importante en este contexto, la de esquema corpóreo. Sobre la presencia de este concepto en la obra merleaupontiana remito al trabajo de mi autoría titulado "La centralidad de la noción de esquema corporal como quiasmo de espacio y movimiento", en: Revista Investigaciones Fenomenológicas, Madrid, v. X (2003), pp. 15-32. Puede verse cómo Merleau-Ponty se ocupa del tema del esquema corpóreo en su Fenomenología de la Percepción, en la p.128 de la versión francesa (Merleau-Ponty, M., Phénoménologie de la Perception, Paris: Gallimard, Collection Tel, 2012) y en la pp. 116ss de la versión castellana (Merleau-Ponty, M., Fenomenología de la Percepción, Cabanes, J. (trad.), Barcelona: Editorial Planeta-Agostini, 1985).

5 Serrano de Haro, A., La precisión del cuerpo. Análisis filosófico de la punteria, Madrid: Trotta, 2007.
}

ARETÉ Revista de Filosofía, vol. XXVIII, Nº 2, 2016 / ISSN 1016-913X 
relacionadas con la adquisición de hábitos corporales que requieren destreza y un importante entrenamiento físico exigen para su descripción (y para su práctica) esa otra dirección mencionada, en especial para dar cuenta de algunos aspectos del proceso de aprendizaje y afianzamiento en la disciplina. El conocimiento del cuerpo físico y los estados del organismo, la aprehensión del espacio geométrico, la "conciencia corporal" de las fuerzas y tensiones, constituyen un momento distinguible en el proceso de adquisición de ciertas destrezas y, a los efectos de su descripción, las alternativas dicotómicas acerca de lo objetivo y lo vivido, de la idea y la experiencia, ocultan dimensiones centrales del fenómeno, tal como se intentará mostrar en el presente estudio.

La comprensión del tema del espacio es emprendida al interior de Fenomenología de la Percepción $n^{6}$ con la intención de aportar elementos descriptivos que permitan aprehender la experiencia perceptiva en toda su complejidad y está planteada en dos dimensiones a las que bien cabría considerar complementarias entre sí. Por un lado, podemos mencionar la relativa a la espacialidad del cuerpo propio y a la relación de esta con el espacio exterior por medio del movimiento; por otro lado, encontramos el tratamiento de lo relativo al mundo perceptivo como campo fenomenal, en el cual se suscita una determinada experiencia del espacio. Ambos análisis tienen en común, como señala Claude Lefort, el afán por "demostrar que el cuerpo solo, y no la conciencia, nos da la clave del problema del movimiento"7. En uno y otro caso el tratamiento de la temática del espacio es abordado con la finalidad de superar las limitaciones explicativas de la concepción intelectualista y de la concepción empirista del espacio, en general, y de la espacialidad del cuerpo, en particular. La reducción intelectualista del espacio a la conciencia, así como la comprensión empirista del espacio como continente no bastan para aprehender, en su peculiaridad, la experiencia del espacio que se da a través del movimiento. En un caso, el intelectualista peca por exceso, ya que es necesaria una operación de coordinación para comprender el espacio; en el otro caso, el empirista peca por defecto, dado que se despoja al espacio de todo componente intencional. Merleau-Ponty, a lo largo de Fenomenología de la Percepción, coloca en el centro de sus análisis "la

\footnotetext{
6 Este trabajo se centrará en el desarrollo de la temática de la espacialidad y el movimiento en el contexto de Fenomenología de la Percepción, por lo que utilizará la versión francesa de la editorial Gallimard de 1945, pero en una reedición en la colección Tel del año 2012, citada en la nota 4. En adelante, las referencias bibliográficas y citas a esta obra serán bajo las siglas $P P$ indicando las páginas de la edición francesa mencionada, seguido de la paginación de la edición castellana indicada en la nota 4 de este trabajo, colocada entre paréntesis, seguido de la página de la versión francesa.

7 Lefort, C., "Le sens de l'orientation", p. 227.
} 
experiencia de...", como un modo de mostrar lo inaprehensible y multiforme de los fenómenos humanos. La descripción de las experiencias del movimiento y del espacio rehúyen toda comprensión que presuponga términos antitéticos y excluyentes (psiquico y físico, sujeto y objeto, inmanente y trascendente). En el contexto de estas críticas, Merleau-Ponty se vale no solo de la experiencia en general de todo agente motriz (con la cual cualquier lector podria identificarse), sino también de la experiencia patológica, con el objeto de revelar aspectos que al sujeto normal le pasan desapercibidos ${ }^{8}$.

De las páginas dedicadas por Merleau-Ponty al tema del espacio, que conciernen de manera directa a la motricidad, me interesa rescatar dos distinciones, tal como se anunció al comienzo, (1) espacialidad de posición y espacialidad de situación y (2) espacio espacializado y espacio espacializante. Ambas distinciones involucran el espacio, mas la primera está referida a la relación del cuerpo con el espacio, mientras la otra concierne a la relación del espacio con el movimiento.

El par posición-situación es la herramienta propuesta en Fenomenología de la Percepción para esclarecer y determinar la clase de espacialidad que caracteriza al cuerpo propio y poder dar cuenta así del fenómeno del movimiento humano, prescindiendo de una conciencia tética de movimiento. La espacialidad de posición, propia de los objetos, establecida en un espacio homogéneo y neutro de coordenadas fijas y bajo el dominio estructurante de una conciencia sin lugar, es contrastada con una espacialidad de situación, la cual le permite a Merleau-Ponty reintroducir el componente intencional de un sujeto encarnado que se encuentra en el mundo "ante sus tareas" en función de proyectos.

La espacialidad de situación, entendida como la peculiar manera de darse el mundo, es considerada por Merleau-Ponty en dos formatos, el del sujeto que padece alguna patología funcional o neurológica y el del agente motriz normal. Para el primero, el mundo se da bajo la forma de algo "ya hecho o fijo", mientras que para el segundo "los proyectos polarizan el mundo" y "hacen aparecer en él como por arte de magia mil signos que conducen la acción, como los letreros de un museo conducen al visitante" 9 . Por un lado, resulta claro que MerleauPonty nos presenta una dicotomía excluyente: el espacio es algo "hecho o fijo"

\footnotetext{
8 Acerca del recurso merleaupontiano a casos patológicos en relación a la comprensión del espacio y la motricidad recomiendo revisar a Jensen, R.T., "Motor Intentionality and the Case of Schneider", en: Phenomenology and Cognitive Science, 2009, pp. 371-388 y a Inverso, H., "Derivas del 'Caso Schneider': Espacialidad, movimiento y reducción fenomenológica en Merleau-Ponty", en: Investigaciones Fenomenológicas, v. XII (2015), pp. 51-70.

9 Merleau-Ponty, M., PP, p. 143 (trad., p.129).
} 
o el "espacio está polarizado por los proyectos de un agente". Por otro lado, la idea de situación, piedra de toque de la concepción merleaupontiana de la motricidad y la acción humanas, postula un cuerpo activo, un "yo puedo". Sin embargo, no parece posible, en este contexto intencional, ponderar o considerar aspectos de índole objetiva tales como el estado fisico y biológico del organismo, las condiciones materiales del entorno, los obstáculos, así como el tamaño o la extensión, entre otros ${ }^{10}$. Merleau-Ponty coloca en términos antitéticos la experiencia de un agente motriz normal y la de un agente motriz enfermo, pero no considera casos como el cansancio, el desánimo, el dolor, la incomodidad, etcétera, los cuales, sin llegar a ser patologías, constituyen modulaciones de la percepción y del movimiento en el sujeto que se presume normal ${ }^{11}$.

Merleau-Ponty nos señala la existencia de una estrecha relación entre los proyectos y las acciones posibles y el espacio que es propuesto por la situación. Tal relación se confirma cuando leemos que las operaciones de proyección, para las cuales el enfermo se encuentra impedido, "exigen un mismo poder de trazar en el mundo dado unas fronteras, unas direcciones, de establecer líneas de fuerza, de componer unas perspectivas, en una palabra, de organizar el mundo dado según los proyectos del momento, de construir en el entorno geográfico un medio (milieu) de comportamiento, un sistema de significaciones que expresa hacia fuera la actividad interna del sujeto"12.

Aquello que el enfermo no puede concretar es, precisamente, lo que en el agente normal se suscita de manera espontánea. Sin embargo, el mundo

\footnotetext{
${ }^{10}$ Como señala Gallagher, el agente en situación no pondera la montaña que intenta escalar o la distancia que se quiere recorrer en términos de cuántos metros de altura o de longitud tiene, sino más bien en cuanto es "escalable", o la meta "alcanzable" ( $c f$. Gallagher, S., "Are Minimal Representations Still Representations?”, en: International Journal of Philosophical Studies, v. XVI, 3 (2008), pp. 351-369). El componente intencional relativiza los aspectos "objetivos" del espacio y el tiempo. ${ }^{11}$ Aquí se podría objetar que Merleau-Ponty sí considera casos semejantes cuando analiza la experiencia de Stratton, en la cual se intenta inducir, mediante el uso de lentes, la inversión del campo perceptivo. Bajo tales condiciones experimentales, el sujeto constata la imposibilidad de moverse en el espacio o realizar acciones de manera espontánea y deberá esperar el paso de las horas y el consecuente acostumbramiento a la nueva situación para volver a disponer de su agencia. En mi opinión, sin embargo, el ejemplo de Stratton ofrece una situación ficticia, artificial, la cual, si en alguna medida contribuye al esclarecimiento del tema, lo hace nuevamente colocando el énfasis en la noción de situación entendida como medio contextual, pues lo que describe (con la intención de explicar) es que el restablecimiento de la posición "vertical" va a depender estrechamente de la capacidad del agente encarnado para comprometerse una vez más en sus proyectos motores, es decir, en su capacidad para convertir lo extraño en familiar. Por este motivo, podemos señalar que aquí la noción de situación no se explica por el experimento, sino, más bien, que el mismo experimento parece estar preparado en función de la noción de situación. Ahora bien, tal como intentaré mostrar, la noción de postura que me interesa introducir proporciona una herramienta descriptiva para el peculiar fenómeno de dominar lo extraño sin volverlo familiar.

12 Merleau-Ponty, M., PP, p. 143 (trad., p.129).
} 
fijo de coordenadas no constituye un límite exclusivo para el enfermo, pues, como se ha mencionado antes, situaciones de cansancio o de dolor pueden restablecer, en el agente motriz, una sensación de extrañeza o incomodidad con el entorno. Las descripciones que Merleau-Ponty realiza del espacio no dan cuenta de la relación en sentido inverso, es decir, la que va del espacio objetivo al agente motriz y sus proyectos. Esto se explica porque su preocupación consiste, más bien, en mostrar cómo un agente encarnado organiza un mundo, cómo se construye un medio contextual de comportamiento sobre el marco geográfico que proporciona el espacio objetivo. Esto implica que, en el curso del análisis, el espacio objetivo desaparece del horizonte de problematización, y solo es mencionado como el correlato negativo del espacio fenomenalmente concebido. Será preciso, pues, avanzar en el desarrollo de su Fenomenología de la Percepción para llegar a las investigaciones acerca del mundo percibido (Segunda Parte), en donde se traza la distinción entre el espacio espacializante (geométrico) y el espacio espacializado (físico), que subyace al ejemplo del museo y a la postulación de proyectos que polarizan el mundo, para volver a encontrar una tematización del espacio objetivo, del espacio geométrico, en vinculación con el espacio fenomenal.

La distinción espacio espacializante-espacio espacializado aparece en Fenomenología de la Percepción como el trasfondo ante el cual Merleau-Ponty se propone contrastar "nuestra experiencia del espacio" exterior. El fenomenólogo francés recurre a esta distinción de raigambre kantiana, presentada por Pierre Lachièze-Rey en "Uso posible del esquematismo kantiano para una teoría de la percepción" (1937) ${ }^{13}$, con la pretensión de superar la definición del espacio en términos de continente dentro del cual las cosas se ubicarian (milieu), y, en contraste, entenderlo como el "poder universal de las conexiones de las cosas", como el medio (moyen) que hace posible que las cosas ocupen un lugar. El espacio espacializado es aquel que experimento viviendo en las cosas, sin reflexión. Se trata del espacio fisico, orientado, que se da según relaciones concretas de derecha-izquierda, arriba-abajo, cercanía-lejanía. Por el contrario, el espacio espacializante es el espacio geométrico cuyas "dimensiones son sustituibles" y posee, según la definición de Lachièze-Rey ${ }^{14}$, potencia para desarrollar el espacio

\footnotetext{
${ }^{13}$ Lachièze-Rey, P., "Uso posible del esquematismo kantiano para una teoría de la percepción", en: Con-Textos Kantianos. International Journal of Philosophy, 1 (2015), pp. 253-258.

${ }^{14} \mathrm{La}$ asunción merleaupontiana del tema de la espacialidad tiene como marco teórico de referencia la comprensión kantiana del espacio, aunque, como bien señala Albizu en la presentación de la traducción del texto de Lachièze-Rey, esta no pueda ser identificada en Fenomenología de la Percepción con ninguna mención textual de fuente. La influencia kantiana está, más bien,
} 
mediante el movimiento del cuerpo ${ }^{15}$. Esto le permitirá a Merleau-Ponty afirmar que el sujeto de la geometría es un sujeto motor y un "yo puedo".

Ahora bien, la noción de espacio espacializante contiene los dos componentes, es decir, lo relativo a la posición y también el carácter intencional, proyectivo y relacional del espacio por medio del movimiento. Se trata de un espacio geométrico, pero a la vez contiene el germen de una comprensión del espacio que puede ser dinamizado mediante el movimiento y el requerimiento de la tarea a realizar. En este sentido podemos decir con Lachièze-Rey que es un espacio intencional. Lo más interesante es, en consecuencia, que mediante la reflexión es posible pasar de una forma del espacio a la otra, pues, sostiene Merleau-Ponty, “...puedo... cuando menos, pensar un puro cambio de lugar que no modificaría en nada al móvil y por consiguiente una pura posición distinta de la situación del objeto en su contexto concreto" ${ }^{16}$. El pasaje de lo vivido a la reflexión significa, en principio, que es posible para el agente motriz recoger las certezas encarnadas (habitualidades) que lo animan acerca de su cuerpo y del espacio para calcular, medir, ponderar, especular, etcétera.

Sin pretender abundar en descripciones harto conocidas, me limitaré, a continuación, a señalar algunos puntos centrales de la concepción merleaupontiana del espacio del cuerpo vivido o fenomenal, con el objetivo de mostrar por qué entiendo que una noción como la de postura puede constituir un aporte significativo para la comprensión de los fenómenos relativos a la motricidad humana.

La fenomenología merleaupontiana de la corporeidad en el contexto de Fenomenología de la Percepción se concentra en la descripción del cuerpo vivido con el propósito de develar la experiencia que pone de manifiesto las limitaciones de la concepción objetiva del cuerpo. En función de esto y mediante la

mediada por la lectura y exégesis de Lachièze-Rey, quien en el texto de 1937, presenta la distinción entre el espacio espacializado y el espacio espacializante, con la intención de esclarecer el problema de la localización en la teoría de la percepción del filósofo de Königsberg. Para MerleauPonty esta distinción es solo un momento a superar en busca de una tercera forma del espacio, la relacionada con nuestra experiencia. No obstante, señala que esa experiencia fenomenal del espacio, protagonizada por el cuerpo propio, es algo que está presupuesto ya en el mismo análisis kantiano. Podria decirse entonces que el cuerpo pasivo (así como los objetos) subyace al espacio espacializado, mientras que el cuerpo en cuanto potencia intencional y motriz subyace al espacio espacializante ( $c f$. Merleau-Ponty, M. PP, p. 295 (trad. p. 363)).

15 "...o bien no reflexiono, vivo en las cosas y considero vagamente el espacio, unas veces como el medio (milieu) de las cosas, otras veces como su atributo común, o bien reflexiono, retomo (ressaisis) el espacio en su origen (source), pienso actualmente las relaciones que hay debajo de esta palabra, y me percato luego de que estas solamente viven gracias a un sujeto que las describe y que las lleva; paso del espacio espacializado al espacio espacializante" (ibid., p. 291 (trad. p. 258)). ${ }_{16}$ Ibid., p. 291 (trad. p. 259). 
descripción del fenómeno motriz, Merleau-Ponty introduce la distinción entre posición y situación que le permite, en primer lugar, diferenciar la espacialidad del cuerpo vivido de la de los objetos en el espacio en general del espacio objetivo; en segundo lugar, reformular la definición cartesiana del espacio de coordenadas fijas; y por último, postular una concepción original del espacio que se encuentra subordinada al movimiento antes bien que a la conciencia.

Las precisiones en torno al espacio del cuerpo propio o vivido son planteadas en relación con el esquema corporal, el cual es presentado como la organización dinámica del cuerpo ante sus tareas, ante los proyectos motrices que lo solicitan y por esta razón, no puede ser entendido en términos de asociación o sintesis de representaciones cinestésicas ni tampoco como un todo, ya sea principio o estructura, que precede a las partes. Por el contrario, el esquema corpóreo aparece en estas páginas como un sistema práxico (prenoético y pretético) en cuya conformación se encuentra implicada la estrecha vinculación del sujeto con el mundo. Teniendo en cuenta esta definición del esquema corpóreo, Merleau-Ponty avanza en la caracterización de la espacialidad del cuerpo propio o vivido con un importante énfasis en su dimensión kinética. A diferencia de los objetos exteriores o las "sensaciones espaciales", los cuales se hallan dotados de una espacialidad de posición, el cuerpo propio habita una espacialidad de situación ${ }^{17}$, la cual -antes bien que un "lugar" determinado en un espacio organizado según coordenadas fijas y cuya existencia depende de una conciencia capaz de aprehender ese espacio- consiste en un "saber absoluto" de la ubicación espacial del cuerpo propio, del entorno, como también de la orientación y de la postura en que se encuentra el agente ante sus tareas ${ }^{18}$. La espacialidad de posición puede ser representada como un tablero de juego de damas donde los objetos se ubican de manera indistinta y el movimiento solo se percibe como cambio de lugar. Por el contrario, afirma Merleau-Ponty, "la palabra 'aquí' aplicada a mi cuerpo no designa una posición determinada con respecto a otras posiciones o con respecto a unas coordenadas exteriores, sino la instalación de las primeras coordenadas, el anclaje del cuerpo activo en un objeto, la situación del cuerpo ante sus tareas"19.

\footnotetext{
${ }^{17}$ Ibid., p. 129 (trad. p. 117).

18 "Si, de pie delante de mi mesa, me apoyo en ella con mis dos manos, solamente estas quedarán acentuadas y todo mi cuerpo seguirá tras ellas como una cola de cometa. No es que yo ignore la ubicación de mis hombros o de mi zona lumbar, sino que esta queda envuelta en la de mis manos y toda mi postura se lee... en el apoyo que estas toman sobre la mesa" (ibid., p. 129 (trad. p. 117)).

${ }_{19}$ Ibid., p. 130 (trad. p. 117).
} 
La noción de espacialidad de situación constituye un interesante aporte porque no circunscribe la definición del espacio a determinaciones meramente físicas, geométricas o biológicas. Podemos decir, más bien, que supera esas dimensiones sumando a su comprensión un componente antropológico e intencional provisto por el agente encarnado, cuando se instituye como punto de vista. El espacio comprendido en términos de situación no es extensión, no es distancia entre los cuerpos, no es parte, tampoco es hábitat, es más bien el sistema formado por el entrecruzamiento, del espacio corpóreo y el espacio exterior.

La potencia descriptiva de la noción de situación consiste, precisamente, en que permite dar cuenta del "paso" del espacio objetivo a un espacio pleno de significaciones, un medio contextual y familiar, en que el agente encarnado realiza (o no) sus proyectos.

Ahora bien, una vez instalados en ese espacio vivido, ¿qué posibilidad existe de volver a recoger nuevamente ese fondo que el espacio objetivo ofrece y que en algunos fenómenos motrices se hace necesario recuperar (ya sea porque se han modificado las circunstancias del cuerpo que se mueve o en el cuerpo que se mueve) para instalar nuevos proyectos?

En la descripción merleaupontiana del espacio por medio del movimiento no parece haber lugar para ese retorno ${ }^{20}$. Merleau-Ponty pretende, mediante el recurso a la estructura figura-fondo (en la cual el cuerpo constituye el tercer término sobrentendido que tiene como doble horizonte el espacio exterior y el espacio corpóreo), relegar la descripción del movimiento a ese espacio convertido en medio contextual para el comportamiento, medio familiar y pleno de habitualidades, irreductible al espacio geométrico. Frente a esto, el espacio objetivo no es para Merleau-Ponty más que una explicitación conceptual, una tematización del espacio orientado, vivido, aunque desprovisto de todo componente fenomenal ${ }^{21}$.

\footnotetext{
${ }^{20} \mathrm{Y}$ cuando tal retorno se da, es debido a alguna patología como las sufridas por Schneider o como consecuencia de la reflexión con el objetivo de someter al espacio a alguna clase de análisis. ${ }^{21}$ Sobre el origen del espacio objetivo como un producto derivado del espacio vivido y la discusión al respecto, recomiendo Merleau-Ponty, M., PP., p. 131 (trad. p. 118) en las páginas dedicadas al espacio orientado. Merleau-Ponty desarrolla, además, las ideas respecto de la génesis del espacio vivido en la segunda parte de la Fenomenología de la Percepción, en particular cuando se refiere a la constitución de los niveles espaciales como una manera de superar las alternativas explicativas del intelectualismo y del empirismo de experiencias como las de Stratton o Wertheimer. En ese contexto, afirma que no es posible disociar el "ser (estar) del ser (estar) orientado" (ibid., p. 301 (trad. p. 268)) $\mathrm{y}$, a continuación agrega, "cada uno de los niveles en los que vivimos aparecen uno tras otro cuando echamos ancla en un 'medio' (milieu) que se nos propone. Este medio no es espacialmente definido más que para un nivel previamente dado. Así la serie de nuestras experiencias, hasta la primera, se transmiten una espacialidad ya adquirida" (Merleau-Ponty, M., ibid., p. 302 (trad. p. 268)).
} 
Si bien concuerdo plenamente con esta concepción de la génesis del espacio vivido y reconozco el aporte que ha constituido para la comprensión del movimiento como anclaje y posibilidad kinética, entiendo también que el fenómeno del movimiento se vuelve todavía más complejo cuando lo que se intenta describir es la experiencia de agentes entrenados y diestros que se enfrentan a desafios físicos de gran envergadura (escalar, bailar, correr, nadar, etcétera). Estos casos parecen requerir nuevas herramientas que, por un lado, tengan en cuenta el componente fenomenal del movimiento y del espacio, pero que permitan, además, abordar fenómenos en los cuales el espacio geométrico se revela y cobra densidad ontológica, es decir, que adquiere una presencia efectiva en la propia experiencia del agente encarnado, deja de ser una construcción categorial para transformarse en una posibilidad (o en un obstáculo, según sea el caso) de movimiento y acción.

\section{Postura y motricidad}

“Dibujar a María haciendo 'el puente' era igual que dibujar a un minero trabajando en una veta muy angosta. El cuerpo de Maria era muy femenino, pero en su experiencia visible del esfuerzo y la resistencia parecía comparable al del minero.... Su dualidad se hacía evidente en su quietud -el pie izquierdo completamente relajado descansaba en el suelo como un animal dormido- y en el conjunto de vectores de sus caderas y de su espalda preparadas para hacer frente a cualquier peso muerto."

(El cuaderno de Bento, John Berger)

Los estudiosos de Merleau-Ponty contamos con el importante logro teórico que significó la superación, en el análisis del tema del espacio (en general) y de la motricidad humana (en particular), de la espacialidad de posición por la espacialidad de situación y de la dicotómica comprensión del espacio en términos de espacio exterior, en el que se posicionan los objetos, y espacio corporal que "está de mi lado". La redefinición de la relación entre estos dos espacios en términos de "sistema práctico"22 posibilitó incluso una reconfiguración del estatuto de los objetos, en la medida en que pasaban de ser cosas autónomas para convertirse en polo o blanco de mi acción ${ }^{23}$. Sin embargo, entiendo que la

22 Ibid., p. 132 (trad. p. 119).

23 Afirma Merleau-Ponty: "Puedo instalarme, por medio de mi cuerpo, como potencia de cierto número de acciones familiares en mi entorno como conjunto de manipulanda, sin apuntar a mi cuerpo ni a mi entorno como objetos en sentido kantiano, es decir, como sistemas de cualidades ligadas por una ley inteligible, como entidades transparentes, libres de toda adherencia local o 
distinción posición-situación, tan fructífera en los análisis merleaupontianos, puede volverse un recurso explicativo dogmático si se lo adopta de manera acrítica y no se reflexiona acerca de su alcance e implicancias. Cuando se intenta profundizar en su poder descriptivo, en su capacidad para dar cuenta de ciertos fenómenos relativos al movimiento que conciernen a sujetos diestros bajo circunstancias especiales ${ }^{24}$, salen a la luz ciertas limitaciones que obligan su revisión. Esta empresa no implica una puesta en tela de juicio de tan lúcida y valiosa distinción, por el contrario, constituye un llamado de atención con la finalidad de restablecer el valor conceptual de esa herramienta al cual propongo complementar con el aporte de la noción de postura.

La espacialidad de situación le sirve a Merleau-Ponty para dar cuenta del sujeto encarnado ante sus proyectos. En ese contexto, precisamente y tal como ya ha sido mencionado, aparece una alusión a la noción de postura en relación con la noción de esquema corpóreo. Ahora bien, qué sucede cuando se irrumpe la habitualidad, cuando se corta el flujo de proyectos y respuestas que la solicitud del mundo había instituido. Merleau-Ponty nos dirá que el cuerpo tiene la capacidad de restituir esa familiaridad, generando un nuevo vínculo con el medio, y lo justifica al afirmar que “... mi cuerpo se me aparece como postura en vista de una cierta tarea actual o posible", pues "su espacialidad no es como la de los objetos exteriores o como la de las 'sensaciones espaciales', una espacialidad de posición, sino una espacialidad de situación” 25.

Si bien hay una clara alusión a una noción de postura, en el párrafo citado, esta no coincidiría con la acepción que estamos intentando precisar aquí. Encuentro que la noción que vincula la postura al esquema corpóreo ${ }^{26}$ permanece todavia atada a las limitaciones señaladas respecto de la espacialidad de situación y, si bien Merleau-Ponty contempla en su significación la posibilidad de asumir nuevas actitudes corporales y motrices, ellas se encuentran determinadas por el medio y las tareas. Por otro lado, la noción de postura merleaupontiana, al definirse en ese contexto, es reluctante a toda referencia a una espacialidad objetiva o geométrica. Merleau-Ponty conserva así una perspectiva dicotómica

temporal y prestas para la denominación o, cuando menos, para un gesto de designación" (ibid., p. 135 (trad. p. 122)).

${ }^{24}$ Aquí hago referencia a casos como el del deportista, el bailarín o el performer, para quienes el movimiento se desarrolla en un medio que, en principio, no es familiar e incluso muchas veces nunca llega a serlo, pues se cambia regularmente de ubicación (el campo de deportes, o el escenario). ${ }^{25}$ Ibid., p. 129 (trad. p. 117).

${ }^{26}$ Por otro lado, es importante mencionar que Merleau-Ponty está influenciado por el trabajo de H. Head, para quien el esquema corporal es un esquema postural. 
de la espacialidad que tiene por efecto una concepción univoca y unidireccional de la relación del espacio y el cuerpo. La noción merleaupontiana de postura es un cifrado del movimiento esperado, requerido por la tarea y, por ese motivo, contribuye a la restitución de la habitualidad. Sin embargo, tal como es comprendida por Merleau-Ponty, parecería no bastar para explicar un cambio repentino e inesperado de las circunstancias motrices y, la consecuente urgencia que se suscita, de "adoptar" nuevas actitudes motrices provocadas por la emergencia del espacio objetivo, ya sea por cansancio, tensión muscular o dolor. Lo que intento mostrar es que detrás de la noción de situación, en el contexto de la cual adquiere significación la "postura" merleaupontiana, todavía subyace un componente de pasividad heredada de la concepción del agente como móvil antes bien que como moviente, es decir, como sujeto de movimiento, en la medida en que el movimiento es analizado en términos de habitualidades.

La noción merleaupontiana de postura permite describir un estado de cosas en una situación determinada, pero no alcanza para dar cuenta de un cambio repentino en la situación que requeriria una toma (incluso inesperada) actitud motriz.

Esto no significa afirmar que las limitaciones de la noción merleaupontiana de postura residan en la caracterización del cuerpo como tendencia a restituir la habitualidad. El problema se origina más bien, en mi opinión, en la postulación de la noción de situación como contexto a partir del cual se define la postura. En la situación, el agente se encuentra alojado, está en ella; la situación es algo que sobreviene una vez que se ha producido el anclaje espacial del cuerpo habitual. Esto no excluye, por cierto, la posibilidad práctica y concreta de la transformación de la situación por obra del sujeto (por ejemplo, un cambio de planes). Sin embargo, en la descripción de la situación, el agente motriz y sus posibilidades de movimiento, se verían condicionados por ese entorno conformado por el encuentro de cuerpo, mundo y tareas. En consecuencia, en el intento por comprender el movimiento, se suscitaría una suerte de subordinación del agente a los proyectos, metas y fines que lo determinan, antes bien que la posibilidad inversa de reconocer (al menos en algunos casos) al movimiento mismo como origen de esos proyectos.

La noción de situación, en mi opinión, corre el riesgo de anquilosar el estudio del movimiento cuando: 1) su uso oculta cierta sobredeterminación explicativa de la solicitud del mundo por sobre el agente ${ }^{27}$ y 2) no se explicita

\footnotetext{
${ }^{27}$ Para justificar esta afirmación es necesario realizar un rodeo que nos permita captar la noción merleaupontiana de situación en toda su magnitud. Si bien, como se intentó mostrar, esta noción 
la relación entre el espacio objetivo, el agente encarnado y el componente intencional del movimiento, es decir, la relación entre la ponderación de las dimensiones del espacio o de la medida de mi cansancio para reinstaurar la relación intencional con el mundo.

$\mathrm{Si}$, entonces, lo que nos interesa explicar mediante el uso de la noción de situación es, entre otras cosas, cómo aparece el espacio vivido, debemos poder dar cuenta también de la manera en que el movimiento se relaciona con las coordenadas objetivas, con esa dimensión geométrica que luego queda subordinada y oculta en la experiencia del espacio.

En orden a superar los obstáculos que presenta el uso acrítico de la noción de situación y hacer explícito aquello que, según entiendo, Merleau-Ponty deja sin explicar, es que propongo sumar a la descripción del fenómeno del movimiento humano otra noción de postura. Introducir una nueva noción de postura no tiene como objetivo sustituir la noción de situación, sino más bien ofrecer un complemento, que permita dar cuenta de la relación del agente con sus proyectos, distinguir el movimiento "motivado y dirigido" del movimiento "situado y orientado" y ponderar qué significa y cuál es el alcance de la adopción de una postura.

Imaginemos dos situaciones: me despierto en medio de la noche, la espacialidad de los objetos se me ofrece como un recuerdo sedimentado de aquello que ha sido percibido infinidad de veces durante la vigilia, estoy en situación y necesito trasladarme por la habitación en busca de un vaso de agua. El movimiento a tientas hace aparecer signos, como dice Merleau-Ponty, que van "guiando" el recorrido, el espacio geométrico, desaparece bajo las determinaciones del espacio físico, espacializado, apelamos al espacio vivido. Por lo general, en una situación como esa no recurro a la conciencia de que la habitación tiene cuatro metros cúbicos o que está orientada hacia el oeste. La acción en este caso es conducida, guiada, según lo presenta Merleau-Ponty en Fenomenología de la Percepción. Los interrogantes que surgen en consecuencia son: quién conduce la acción y qué es lo que la guía. Sería un error intentar buscar la

aparece en Fenomenología de la Percepción en relación con el tema del espacio, su campo semántico no se circunscribe a ese contexto. Por el contrario, se trata de una herramienta de la cual se vale Merleau-Ponty incluso para referirse a la libertad humana. Quizá el sentido que refleje de manera más fehaciente el propósito de este trabajo es el aludido en relación con el miembro fantasma y el rechazo de la deficiencia que alli se pone de manifiesto. La experiencia patológica irrumpe en lo que Merleau-Ponty caracteriza como "mundo completo", el cual, aun ante la falta de un miembro, continua insinuándose al sujeto como un entorno de objetos manipulables o pleno de proyectos realizables (cf. ibid., p. 111 (trad. p. 100)). Se ve expresado con claridad en esas páginas que el entorno con sus proyectos determina y condiciona al sujeto. 
respuesta ya sea de un lado, el del agente encarnado, o del otro, es decir, el de la situación espacial ofrecida. Los análisis merleaupontianos nos han enseñado que es en el entrecruzamiento de cuerpo y mundo, de agencia y espacio, que se deben buscar las respuestas a esa clase de preguntas. Esto significa que el "cuerpo en movimiento" y los "proyectos motores" son inseparables, pues hay una relación reciproca entre situación y movimiento, y la orientación espacial es dependiente de la relación antes bien que de uno u otro componente aislado.

Ahora bien, ¿qué sucede cuando, aquello que queremos describir es el movimiento dirigido y de máxima precisión, realizado por un agente entrenado, antes bien que el movimiento patológico o el cotidiano de un agente normal?

Imaginemos una situación semejante a la antes mencionada, pero que sucede bajo otras circunstancias, por ejemplo, un mareo momentáneo. Presa del mareo necesito recorrer la habitación, sin embargo, en este caso ni mi condición encarnada ni el espacio me ofrecen certidumbres, sino que para poder movilizarme tengo que poder encontrar la posición vertical y mantenerme en ella. En este caso se vuelve necesario un "cierto cálculo", algún tipo de "ponderación" o "mensura" del espacio para poder emprender el recorrido. En ocasiones como esta parece requerirse aquello que describía Merleau-Ponty como el paso del espacio espacializado al espacio espacializante, es decir, a la reflexión acerca del espacio. El espacio espacializante es susceptible de ser descrito, sus dimensiones son sustituibles debido a que se trata de un espacio homogéneo e isótropo y se piensa en términos de lugar y posición.

Ahora bien, en el último ejemplo me interesa poner en evidencia que hay ciertas experiencias motrices y del espacio en las cuales el llamado espacio objetivo, las coordenadas geométricas, no son algo que deba quedar solapado, envuelto, por la vivencia del espacio vivido, pues, por el contrario, adquiere densidad y su presencia se impone al propio movimiento.

En orden a subsanar las limitaciones mencionadas de las descripciones del movimiento y la concepción del espacio, considero necesario complementar los análisis merleaupontianos con una comprensión de la espacialidad que, además, de la situación, permita incorporar algunos elementos (llamémosle) “objetivos" y/o geométricos. Podemos contar entre ellos, por caso, todo lo relativo al estado y condición del organismo ${ }^{28}$, su voluntad motriz (la cual no se reduciría de manera exclusiva a los proyectos que nos solicitan, sino también

\footnotetext{
${ }^{28} \mathrm{Y}$ por esto debería entenderse no solo las condiciones fisiológicas en que se encuentra sino también las disposiciones con que se prepara (atención, tensión o relajación de los músculos, respiración, concentración).
} 
a la disposición física para movernos) y, fundamentalmente, el espacio espacializante, y junto con él los elementos que hacen posible la "dirección" del movimiento. Estos condicionamientos, bajo los cuales se pueden dar también experiencias genuinas del espacio y fenómenos motrices, no cuentan en la descripción fenomenal del espacio y del movimiento.

La noción que en mi opinión cumple con estos requisitos es la de postura tal como es presentada por Agustín Serrano de Haro en su notable estudio ya mencionado. El autor concentra en esa obra sus descripciones de la postura corporal en relación con el fenómeno del apuntar; no obstante, entiendo que sus observaciones pueden hacerse extensivas a fenómenos de motricidad ordinaria en los cuales prevalece la precisión y direccionalidad del movimiento ${ }^{29}$. Por otro lado, considero que tomar las herramientas descriptivas de Serrano de Haro es una manera de aceptar el desafio suscitado en la crítica de Maxine SheetsJohnstone a Merleau-Ponty, cuando afirma que en sus análisis falta el estudio del movimiento desempeñado por agentes motrices diestros y entrenados ${ }^{30}$.

Serrano de Haro se vale de la distinción entre posición y postura, la cual, a los ojos del lector merleaupontiano, parece aludir de alguna manera a la distinción entre una espacialidad de posición y una espacialidad de situación. La posición, dirá el autor en relación al fenómeno del apuntar y consecuente lanzar, se encuentra "determinada ante todo por la distancia efectiva respecto del blanco"31. La posición remite así a un espacio objetivo en el cual se ubican el agente, el útil y el blanco y, podemos decir, que esta forma de espacialidad se encontraría dominada y regida por la posición del objeto/blanco. Las coordenadas espaciales y la orientación de la postura son dependientes del lugar en el que se encuentra la meta del lanzamiento. Como señala Serrano de Haro, la postura está librada a la determinación del sujeto y es "una peculiar 'compostura' del cuerpo como un todo; en ella se fija por tanto la relación en que los distintos miembros corporales se conforman o ajustan entre sí y por tanto la relación en que el cuerpo entero se acomoda al órgano motriz que ha de ejecutar el lanzamiento"32.

\footnotetext{
${ }^{29}$ Esta licencia en la extensión de su noción al ámbito de los movimientos ordinarios no parece reñirse con la voluntad del autor, pues anima al lector a revisar "el hecho vivido tal como él se experimenta en el mundo cotidiano de la vida" teniendo en claro que aquello que interesa es "su estructura interna de sentido tal como puede reconocerla cualquiera que en alguna ocasión haya practicado la punteria" (Serrano de Haro, A., o.c., p. 14).

${ }^{30}$ Cf. Sheets-Johnstone, M., The Corporeal Turn. An Interdisciplinary Reader, Exeter: Imprint Academic, 2009.

${ }^{31}$ Serrano de Haro, A., o.c., p. 27.

${ }^{32}$ Ibid., p. 28.
}

ARETÉ Revista de Filosofía, vol. XXVIII, N² 2, 2016 / ISSN 1016-913X 
Parece estar presente en la "compostura" un componente subjetivo, voluntario podríamos decir, que si bien caracteriza a la postura, no oculta ni desatiende los componentes objetivos de la misma, necesario para el lanzamiento. Ese aspecto es, precisamente, el que suscita mi particular interés. Esta noción de postura no solo no prescinde, sino que, además, otorga relevancia a los aspectos objetivos en la vivencia del espacio. Serrano de Haro señala que el lanzador, antes de realizar el movimiento, relega "a la desatención todo aquello que no es parte objetiva de la situación de lanzamiento, que no tiene que ver con la ubicación del blanco, con el móvil, con la distancia entre ambos, etcétera" ${ }^{33}$, esto significa que en el movimiento de precisión los componentes objetivos (distancia, peso, velocidad, intensidad, tensión, etcétera) juegan un rol fundamental, pues de ellos depende en alguna medida la acción exitosa.

La noción de postura permitiría, en consecuencia, la descripción de fenómenos motrices que no se encuentran de manera completa dominados por el hábito (aun cuando se requiera cierto entrenamiento en el lanzar, por ejemplo, siempre existirán condicionamientos y obstáculos que interrumpen la dialéctica de la habitualidad y que deben poder ser superados con éxito).

Tres aspectos centrales de la noción de postura pueden ser sin inconvenientes sumados a la descripción merleaupontiana del fenómeno motriz. 1) La jerarquización del esquema corpóreo, el cual si bien es una totalidad y hace sistema con el mundo, reclama en ciertos momentos de una organización particularizada. En el caso del lanzamiento la organización del esquema corporal se encuentra jerarquizada en función del miembro móvil (ya sea el brazo o la pierna), en el movimiento dirigido ordinario podemos reconocer también zonas protagónicas (el torso, por ejemplo, al levantarme o la cadera y los miembros inferiores al avanzar). La intrafección relativa a mi cuerpo estaría en esos casos potenciada por la atención sobre determinadas regiones del esquema motriz. 2) Otra característica de la postura es su aparente inmovilidad o, para ganar en precisión, la ausencia de locomoción ${ }^{34}$. Esto lo interpreta Serrano de Haro en términos de "resultante de fuerzas que se mantienen en equilibrio" antes bien que "resultado de cambios de posición relativa entre miembros corporales" 35. El cuerpo, cuando adopta una postura, es todo movimiento, tensión, atención, contracción; cada una de estas manifestaciones motrices puede excluir la "locomoción" pero de ninguna manera el movimiento, pues la postura es

33 Ibid., p. 23.

34 Morris, D., o.c., p. 140.

35 Serrano de Haro, A., o.c., p. 29.

ARETÉ Revista de Filosofía, vol. XXVIII, N², 2016 / ISSN 1016-913X 
precisamente preparación móvil para el movimiento ${ }^{36}$. Por último, 3) resulta importante destacar que lo que Serrano de Haro denomina la "indole carnal del cuerpo" (sensible, afectiva), y aquí hemos entendemos como el "estado del organismo", adquiere en la postura independencia fenomenológica a tal punto que posee relevancia y determinación explicativa. Esto significa que lo que podríamos denominar el particular estado del agente encarnado en situación (el mareo, la incomodidad, el cansancio, el dolor, etcétera) no puede ser subsumido a la situación general, sino que adquiere especificidad, se destaca y diferencia del resto de los componentes de esta y puede, según los casos incluso, imponerse de manera anticipatoria a los proyectos haciéndolos aparecer de determinada manera o provocando una determinada modulación de la empresa perceptivomotriz. Pensemos, por ejemplo, en la actividad de escalar una pendiente: la situación está dominada por el agotamiento en general, los movimientos están determinados por el cansancio (los pies son arrastrados, la respiración agitada, se produce cierta laxitud muscular); sin embargo, puedo, llegado el caso, sobreponerme a ese "estado", adoptar una postura corporal vertical y recuperar el aliento para ganar la cumbre. La postura funcionaria en este caso como una suerte de switch que permite de manera voluntaria transformar una situación determinada (adversa por el cansancio y el desánimo) en una nueva situación (favorable ante el estimulo que significa llegar a la meta).

La noción de switch propuesta puede, sin lugar a dudas, inducir a una comprensión equívoca si se opta por interpretarla como un interruptor antes bien que como un conmutador ${ }^{37}$. La noción de interruptor presupondría la posibilidad de paralizar o interrumpir, como el propio nombre lo indica, el movimiento para introducir una modificación; no obstante, es posible encontrar en la segunda acepción un énfasis en la idea de que cualquier modificación de la situación operada por la postura se produce en el mismo flujo continuo de percepción-movimiento-encarnación. No existiría así una distancia real entre la voluntad de retomar el dominio de mis movimientos, la percepción de la meta y la condición de mi cuerpo; por lo tanto, no se constataría el paso de un "estado" a "otro estado" radicalmente diferenciado. La adopción de la (nueva) postura es así presentada como un momento identificable, aunque no aislable,

\footnotetext{
${ }^{36}$ Como sostiene Morris: "Postura, como preparación para el movimiento, es una relación móvil con el mundo, aun cuando no implica locomoción evidente” (Morris, D., o.c., p. 140).

${ }^{37}$ Según el Diccionario de la Real Academia Española, el conmutador es un dispositivo que poseen algunos aparatos eléctricos y cumple la función de permitir que una corriente cambie de conductor sin verse interrumpida.
} 
ni discreto, en el que una determinada situación y los proyectos que por ella me solicitan se transforman, adquieren nueva forma o se modulan de una determinada manera, la cual es distinguible de la que la precede.

La noción de postura adquiere relieve al funcionar como un complemento descriptivo del par posición-situación, tanto postura como situación permiten dar cuenta de la especificidad propia de la corporeidad humana en la medida en que no son atribuibles a los objetos sino solo al cuerpo vivido. Retomando la cita en la cual Merleau-Ponty afirma que la palabra "aquí" aplicada a mi cuerpo se refiere al anclaje del cuerpo activo, deberiamos agregar que se trata más bien de la expresión "aquí así", es decir, en una determinada postura corporal, lo cual implica, además, el estar abierto a ciertas y determinadas posibilidades motrices.

\section{Bibliografia:}

Barbaras, R., "Motricité et phénoménalité chez le dernier Merleau-Ponty", en: Richir, M. y E. Tassin (eds.), Merleau-Ponty, Phénoménologie et Expériences, Grenoble: Jérôme Millon, 1992.

Barbaras, R., "Perception and Movement: the End of the Metaphysical Approach", en: Evans, F. y L. Lawlor (eds.), Chiasms: Merleau-Ponty's notion of Flesh, Albany: State University of New York Press, 2000, pp. 77-87.

Battán Horenstein, A., "La centralidad de la noción de esquema corporal como quiasmo de espacio y movimiento”, en: Revista Investigaciones Fenomenológicas, 10 (2013), Madrid, pp. 15-32. http://dx.doi.org/10.5944/rif.10.2013.11872

Berger, J., El cuaderno de Bento, Buenos Aires: Alfaguara, 2013.

Dillon, M., Merleau-Ponty's Ontology, Bloomington e Indianapolis: Indiana University Press, 1988.

Gallagher, S., How the Body Shapes de Mind, Nueva York: Oxford University Press, 2005. http://dx.doi.org/10.1093/0199271941.001.0001

Gallagher, S., "Are Minimal Representations still Representations", en: International Journal of Philosohical Studies, v. XVI, 3 (2008), pp. 351-369. http://dx.doi. org/10.1080/09672550802113243

Inverso, H., "Derivas del 'Caso Schneider': Espacialidad, movimiento y reducción Fenomenológica en Merleau-Ponty”, en: Investigaciones Fenomenológicas, 12 (2015), pp. 51-70.

Jensen, R.T., "Motor Intentionality and the Case of Schneider", en: Phenomenology and Cognitive Science, 2009, pp. 371-388. http://dx.doi.org/10.1007/ s11097-009-9122-x

Kristensen, S., "Le mouvement de la création. Merleau-Ponty et le corps de l'artiste", en: Alter. Revue de Phénoménologie, 16 (2008), pp. 243-260. 
Lachièze-Rey, P., "Uso posible del esquematismo kantiano para una teoría de la percepción”, en: Cont-Textos Kantianos. International Journal of Philosophy, 1 (2015), pp. 253-258.

Lefort, C., "Le sens de l'orientation", en: Merleau-Ponty, M., Notes de cours sur L'origine de la geometrie de Husserl. Suivi de Recherches sur la phénoménologie de MerleauPonty, Barbaras, R. (dir.), París: Épiméthée, PUF 1989, pp. 221-238.

Merleau-Ponty, M., Phénoménologie de la Perception, París: Gallimard, Collection Tel, 2012.

Merleau-Ponty, M., Fenomenología de la Percepción, Cabanes, J. (trad.), Barcelona: Editorial Planeta-Agostini, 1985.

Merleau-Ponty, M., Le monde sensible et le monde de l'expression, Ginebra: Mētis Presses, 2011.

Morris, D., The Sense of the Space, Albany: SUNY Press, 2004.

Serrano de Haro, A., La precisión del cuerpo. Análisis filosófico de la puntería, Madrid: Editorial Trotta, 2007.

Sheets-Johnstone, M., The Primacy of Movement, Amsterdam: John Benjamin, 1999. http://dx.doi.org/10.1075/aicr.14

Sheets-Johnstone, M., The Corporeal Turn. An Interdisciplinary Reader, Exeter: Imprint Academic, 2009.

Recibido:

Aceptado: 